\title{
Contributions on highlighting an energy method used for con- structive optimization of the mechanical structure of a mobile robot implemented in humanitarian demining operations
}

\author{
Silviu Mihai Petrişor ${ }^{1}$, Mihaela Simion² \\ 1 Department of Technical Science, “Nicolae Bălcescu” Land Forces Academy of Sibiu, Romania; \\ robmilcap@gmail.com \\ 2 Department of Strength of Materials, Technical University of Cluj-Napoca, Cluj-Napoca, Romania; \\ Mihaela.Simion@rezi.utcluj.ro \\ * Correspondence: robmilcap@gmail.com
}

\begin{abstract}
Modularity in the construction of industrial robots is becoming increasingly popular, as the modules can be assembled in different ways so as to obtain various architectures of industrial robots determined by the requirements of specific applications. Such a robot with modular structure is considered in this paper. The tracked mobile robot, with translation - rotation - translation translation - rotation (TRTTR) modular serial structure, is the subject of national invention patent number RO132301 B1/2021, granted by the State Office for Inventions and Trademarks of Bucharest, to "Nicolae Bălcescu" Land Forces Academy of Sibiu, Romania [1]. The tracked mobile robot has been designed to perform the task of humanitarian demining operations and the mechanical structure, respectively the kinematic analysis were described in detail in papers [1,2]. A first general objective of this paper is to highlight a dynamic-organological method to achieve an architectural structure of mobile robot on tracks, able to replace the human element in areas with high risk for health and life, for detection and demining anti-personnel and anti-armor minefields in countries where military conflicts have taken place. For this scope it was performed the dynamic modeling using the Lagrange's formalism and to achieve the constructive optimization of the TRTTR mechanical structure, in order to obtain a minimum energy consumption through an optimal arrangement of the modules in its structure. A second objective is to improve the action flexibility of the technological product in humanitarian detection and demining operations, as well as for educational purposes, by training highly educated and specialized human resources in the field of advanced military technologies. The differential equations of motion of the robot has obtained and respectively the mechanical design equations, which lead to the optimal choice of the drive motors of the modules in the robot structure. Also, it is presented the mathematical model for obtaining the driving motor of base translation modulus (MTB SIL) of the modular serial tracked robot and the constructive solution of the MTB SIL module.
\end{abstract}

Keywords: robotics, tracked robot, dynamic modeling, constructive optimization, human-artificial partnership, humanitarian demining.

\section{Introduction and preliminary results}

The modular design is based on the realization of interchangeable translation, rotation, tilting and orientation modules with independent drive, positioning and control which, assembled in different ways, lead to various architectures of industrial robots determined by the requirements of specific applications [3]. Thus, being given (n) modules: translation and rotation modules of the robot's arm, respectively a translation module and a rotation module in the arm structure, interconnecting these modules, kinematic variants of industrial robots with 2, 3, 4, 5 or 6 degrees of freedom are obtained [4]. The advantages of modular solutions of industrial robots are: simplification of the structure, compact modules, standardization of components, independent functioning of the modules that form the kinematic chain of the industrial robot, easy and faster assembly process of the 
complete industrial robot. Additional advantages, which is particularly important for manufacturers of industrial robots, are shorter manufacturing times, longer production series and reduced manufacturing costs [5]. In the design stage of industrial robots it is necessary to evaluate some indices, such as: number of degrees of freedom, shape and dimensions of the workspace, mobility, load capacity, coefficient service etc. Such an evaluation is possible only by performing a dynamic study of the robots [6].

Dynamic studies performed on serial robots allow the choice of drive motors, as well as the optimal arrangement of the modules in a modular robot construction so that energy consumption is minimal. The dynamic modeling aims to study the two fundamental problems of the dynamics of industrial robots. In the case of the first problem, also called the direct problem, the laws of variation of coordinates and generalized velocities as a function of time are determined, knowing the mechanical constructive parameters of the robot, the laws of variation of forces and motor moments as a function of time and the initial conditions of the movement of the robot. In the case of the second fundamental problem, also called the inverse problem, the laws of variation according to time of the motor agent (forces and motor moments) are determined if the constructive mechanical parameters of the robot and the laws of variation according to time of generalized coordinates are given. Among the methods of dynamic study of industrial robots are: Lagrange's formalism, the principle of virtual movements in dynamic form, the Newton-Euler method and the iterative method. Obtaining high-performance robots takes into account both the chosen constructive solution and the programming, control, command and control mode. The mass production of a large number of military robots with different architectural structures, acting in workspaces of different shapes and sizes, depending on the requirements of the handling program, can be achieved by designing robot models in modular structure.

The modular design is based on the separate realization of the modules whose construction allows their assembly with other modules [6,8,9]. As a result, various architectures of modular robots, this can be delivered to the beneficiaries in accordance with the requirements of the specific application. Using translation, rotation, tilting and orientation modules, variants of trajectory generating mechanisms from the mechanical structure of modular type robots can be conceived. Following a rigorous dynamic study applied on modular serial robot structures possessing from two to six degrees of freedom, a constructive optimization method can be obtained, according to which the translation and rotation modules can be arranged in a modular robot structure, so that energy consumption is minimal.

In this paper is presented the dynamic modeling and constructive optimization of the mechanical structure of a tracked mobile robot for humanitarian demining operations.

The tracked mobile robot is the subject of national invention patent number RO132301 B1/2021, granted by the State Office for Inventions and Trademarks of Bucharest to "Nicolae Bălcescu" Land Forces Academy of Sibiu, Romania [1,2].

The fundamental objective of the research was the design and practical realization of a technological, robotic and innovative product to carry out the missions of minefields, their total demining and humanitarian demining, in various fields in the time provided for each mission. The operational objectives are the following:

- the robot must perform the full range of activities necessary for the field cleaning process: research, field scanning, marking the corridors made in the mine fields and destroying the mines, in autonomously varied terrain;

- the robot should be built on a low budget, have an increased scanning capability and a demining speed of at least $1.5 \mathrm{~m} / \mathrm{s}$ in slightly rugged terrain, so that it can be used in humanitarian demining;

- to mark the corridors in the minefields throughout the execution of the demining mission;

- the robot should operate on the basis of solar energy using energy-conserving photovoltaic cells to ensure uninterrupted operation, at the normal parameters of the robot, both during the day and at night. 
The principle of choosing the drive motors of the modules from the TRTTR modular serial structure of the tracked robot is presented by using the differential equations of motion resulting from the dynamic modeling, respectively of the organological calculations of the robot. This connects the dynamic equations with the organological ones, which leads to optimal robot architectures in terms of energy consumption.

In this paper it is given as an example the mathematical modeling order to obtain the moment of driving motor of translation modulus of the modular serial tracked robot's base (MTB SIL module). The constructive solution of the MTB SIL module with mechanical components is also presented. As well the advantages, the novelty and the originality of the proposed solution, the technical problem solved by the technological robotic product, the operating mode and the use of the mobile tracked robot in special operations were highlighted.

\section{The dynamic model of the TRTTR modular serial structure of tracked mobile robot}

The tracked robot is composed of the tracked base, with the translation system of the device for unexploded mine detection, and the serial-modular robot of TRTTR type (3 translations: base modulus and vertical and horizontal arm, and 2 rotations: rotation modulus and rotation of clamping device performed by vertical arm) with clamping device (Figure 1.a) [1,2].

Each module in the TRTTR structure is driven by electric motors powered by photovoltaic panels thus ensuring their independent operation and, in case of failure, they can be easily replaced without affecting the operation of the other modules. The modular type structure of the TRTTR robot, as well as the individual supply of the modules lead to the interchangeability of the modules in its structure, obtaining various architectures of robots performing one or more tasks.

For dynamic modeling of the robot the Lagrange's formalism is used. The modules in the robot structure have one degree of freedom, respectively 2 degrees of freedom (vertical arm), and the rotational or translational motion along a specific axis being performed by means of an independently controlled actuator $[9,10]$.

In Figure 1.b is presented the kinematic diagram of the TRTTR modular serial tracked robot and following values has been considered: $l_{i}, i=0 \div 6$ the robot's constructive parameters, $q_{k}, v_{k}, \dot{q}_{k}, \ddot{q}_{k}, \dot{v}_{k}, \ddot{v}_{k}, k=1 \div 5$ the coordinates, generalized velocities and accelerations, $k=1 \div 5$ the number of degrees of freedom, $\bar{F}_{i}, i=1,3,4$ the driving forces, $\bar{M}_{i}, i=$ 2,5 the driving motor moments, $\bar{P}_{i}, i=1 \div 5$ the gravitational forces of the robot's modulus, $m_{i}, i=1 \div 5$ the masses (the own weights) of the robot's modules, $J_{\Delta_{1}}^{(i)}, i=1 \div$ 3 the mechanical moment of inertia relative to the axis $\left(\Delta_{1}\right)$ of the $\left(\mathrm{R}_{1}\right)$ rotation module movable system and of the part of module 3 that is interdependent with module 2, $J_{\Delta_{2}}^{(i)}, J_{O_{i_{1}}}^{(i)}, i=4,5$ the mechanical moment of inertia relative to the $O_{3} z_{3}$ axis of the part of translation module 4 that performs a relative motion along the $\left(\Delta_{2}\right)$ axis and the gripping device while is being handled the object.

The dynamic equations of the TRTTR modular serial structure of tracked mobile robot were deduced by using Lagrange's equation of the second kind, written in the following form:

$$
\frac{d}{d t}\left(\frac{\partial E_{c}}{\partial \dot{q}_{k}}\right)-\frac{\partial E_{c}}{\partial q_{k}}=Q_{k}, k=1 \div 5
$$

where: $E_{c}$ represents the kinetic energy of the robot and $Q_{k}$ the generalized forces. 


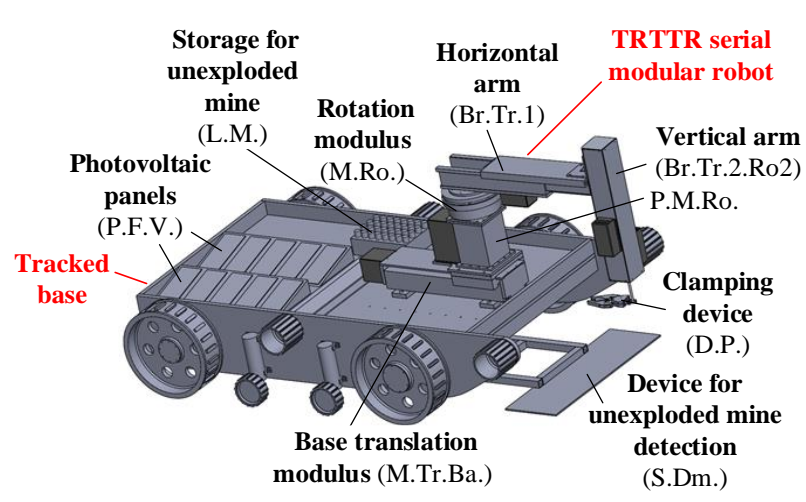

a)

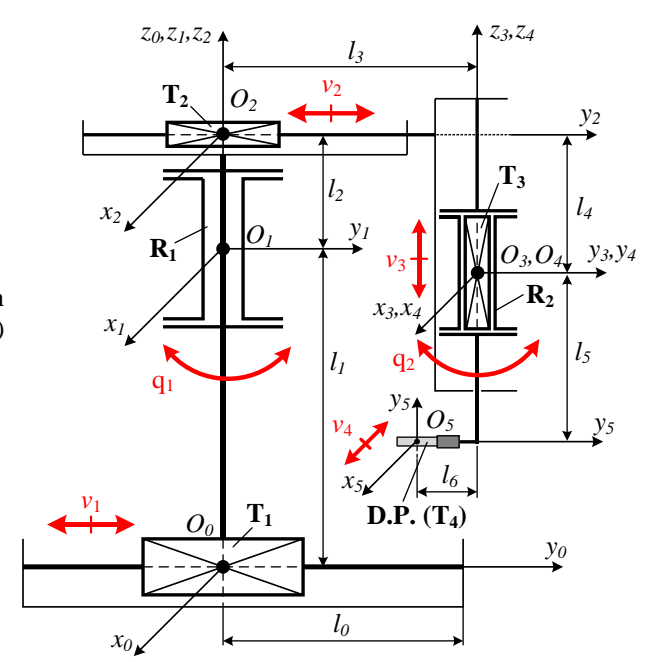

b)

Figure 1. a) Tracked mobile robot - CAD model; b) The kinematic diagram of the TRTTR modular serial tracked robot $[1,2]$.

In order to simplify the calculus, in the dynamic analysis of the robot it was considered that each module of the robot, in the reference system have the origin in the center of the masses, meaning that $x_{c}=y_{c}=z_{c}=0$ and the Cartesian reference systems interdependent with robotic elements were chosen so that they coincide with the main inertia directions corresponding to the system's origin. In this case, centrifugal mechanical moments of inertia are null $\left(J_{x y}=J_{x z}=J_{z y}=0\right)$ and (2) were written under the following form:

$$
E_{c_{i}}=\frac{1}{2} M_{i}\left(v_{x}^{2}+v_{y}^{2}+v_{z}^{2}\right)_{i}+\frac{1}{2}\left(J_{x} \omega_{x}^{2}+J_{y} \omega_{y}^{2}+J_{z} \omega_{z}^{2}\right)_{i}
$$

The kinetic energies, corresponding to the robotic modules type TRTTR, can be consecutively obtained, starting at the robotic base. Thus, for translation module 1 , given that $v_{x 1}=v_{z 1}=0 ; v_{y 1}=\dot{v}_{1}$ the kinetic energy was expressed using the equation:

$$
E_{c_{1}}=\frac{1}{2} m_{1}\left(v_{x 1}^{2}+v_{y 1}^{2}+v_{z 1}^{2}\right) ; \quad E_{c_{1}}=\frac{1}{2} m_{1} \dot{v}_{1}^{2}
$$

In the case of rotation module 2 of the arm, the components of the instantaneous angular velocity $\bar{\omega}_{2}$ and instantaneous velocity $\bar{v}_{02}$ along the $O_{1} x_{1} y_{1} z_{1}$ motion system axes can be specified, namely: $\omega_{x 2}=\omega_{y 2}=0 ; \omega_{z 2}=\dot{q}_{1} ; v_{x 2}=v_{y 2}=0 ; v_{z 2}=\dot{v}_{1}$, as well as the mechanical moment of inertia $J_{z 1}=J_{\Delta_{1}}^{(2)}$, relative to $\left(\Delta_{1}\right)$ rotation axis. The kinetic energy of module 2 has the following equation:

$$
E_{c_{2}}=\frac{1}{2} m_{2} \dot{v}_{1}^{2}+\frac{1}{2} J_{\Delta_{1}}^{(2)} \dot{q_{1}^{2}}
$$

The translation motion of the module 3 , at a given time, was characterized by the following kinematic parameters $\omega_{x 3}=\omega_{y 3}=0 ; \omega_{z 3}=\dot{q}_{1}$ and the kinetic energy and absolute velocity of module 3 were given by the following equation, according to Figure $1 \mathrm{~b}$ :

$$
\begin{gathered}
E_{c_{3}}=\frac{1}{2} J_{z 3} \dot{q}_{1}^{2}+\frac{1}{2} m_{3}\left(v_{x 3}^{2}+v_{y 3}^{2}+v_{z 3}^{2}\right) \\
E_{c_{3}}=\frac{1}{2} J_{z 3} \dot{q}_{1}^{2}+\frac{1}{2} m_{3} v_{03}^{2} ; \quad \bar{v}_{03}=\dot{\bar{v}}_{1}+\dot{\bar{q}}_{1} \times \bar{r}_{3}+\dot{\bar{v}}_{2}
\end{gathered}
$$

The square of $\bar{v}_{03}$ velocity has the equation: 


$$
\bar{v}_{03}^{2}=\dot{v}_{1}^{2}+\dot{v}_{2}^{2}+\dot{q}_{1}^{2}\left(l_{3}+v_{2}\right)^{2}+2 \dot{v}_{1} \dot{v}_{2} \cos q_{1}-2 \dot{v}_{1} \dot{q}_{1}\left(l_{3}+v_{2}\right) \sin q_{1}
$$

and according to perpendicularity relation:

$$
r_{3}=l_{3}+v_{2}, \dot{\bar{v}}_{2} \cdot\left(\dot{\bar{q}}_{1} \times \bar{r}_{3}\right)=0, \dot{\bar{v}}_{2} \perp\left(\dot{\bar{q}}_{1} \times \bar{r}_{3}\right)
$$
equation:

Thus, knowing that $J_{z 3}=J_{\Delta_{1}}^{(3)}$ the kinetic energy of the module has the following

$$
\begin{aligned}
E_{c_{3}}=\frac{1}{2} m_{3}\left(\dot{v}_{1}^{2}+\right. & \left.\dot{v}_{2}^{2}\right)+\frac{1}{2}\left[\left[J_{\Delta_{1}}^{(3)}\right]+m_{3}\left(l_{3}+v_{2}\right)^{2}\right] \dot{q}_{1}^{2} \\
& +m_{3}\left[\dot{v}_{1} \dot{v}_{2} \cos q_{1}-\dot{v}_{1} \dot{q}_{1}\left(l_{3}+v_{2}\right) \sin q_{1}\right] .
\end{aligned}
$$

The movement of the translation module 4 , at a given time, was characterized by the following kinematic parameters: $\omega_{x 4}=\omega_{y 4}=0 ; \omega_{z 4}=\dot{q}_{2}$ the kinetic energy and absolute velocity of module 4 were given by the following equations (Figure 1.b):

$$
\begin{gathered}
E_{c_{4}}=\frac{1}{2} J_{z 4} \dot{q}_{2}^{2}+\frac{1}{2} m_{4}\left(v_{x 4}^{2}+v_{y 4}^{2}+v_{z 4}^{2}\right) \\
E_{c_{4}}=\frac{1}{2} J_{z 4} \dot{q}_{2}^{2}+\frac{1}{2} m_{4} v_{04}^{2} ; \quad \bar{v}_{04}=\dot{\bar{v}}_{2}+\dot{\bar{q}}_{2} \times \bar{r}_{4}+\dot{\bar{v}}_{3}
\end{gathered}
$$

The square of $\bar{v}_{04}$ velocity has the equation:

$$
\bar{v}_{04}^{2}=\dot{v}_{2}^{2}+\dot{v}_{3}^{2}+\dot{q}_{2}^{2}\left(l_{4}+v_{3}\right)^{2}+2 \dot{v}_{2} \dot{v}_{3} \cos q_{2}-2 \dot{v}_{2} \dot{q}_{2}\left(l_{4}+v_{3}\right) \sin q_{2}
$$

and according to perpendicularity relation:

$$
r_{4}=l_{4}+v_{3}, \dot{\bar{v}}_{3} \cdot\left(\dot{\bar{q}}_{2} \times \bar{r}_{4}\right)=0, \dot{\bar{v}}_{3} \perp\left(\dot{\bar{q}}_{2} \times \bar{r}_{4}\right)
$$

Thus, knowing that $J_{z 3}=J_{O_{4}}^{(4)}$ the kinetic energy of the module has the following equation:

$$
\begin{gathered}
E_{c_{4}}=\frac{1}{2} m_{4}\left(\dot{v}_{1}^{2}+\dot{v}_{2}^{2}+\dot{v}_{3}^{2}\right)+\frac{1}{2}\left[J_{\mathrm{O}_{4_{1}}}^{(4)}+m_{4}\left(l_{4}+v_{3}\right)^{2}\right] \dot{q}_{2}^{2}+ \\
+m_{4}\left[\dot{v}_{2} \dot{v}_{3} \cos q_{2}-\dot{v}_{2} \dot{q}_{2}\left(l_{4}+v_{3}\right) \sin q_{2}\right]
\end{gathered}
$$

The movement of the rotation (orientation) module 5 , at a given time was characterized, by the following kinematic parameters $\omega_{x 5}=\dot{q}_{1} \sin q_{2}, \omega_{y 5}=\dot{q}_{1} \cos q_{2}, \omega_{z 5}=\dot{q}_{2}$, and for the determinate the velocity of the center of gravity afferent to module $5, \bar{v}_{05}$, in (13) only change that appears is to replace the position vector $\bar{r}_{4}$ with $\left|\bar{r}_{5}\right|=l_{5}$. Knowing also that $J_{z 4}=J_{\Delta_{2}}^{(5)}, J_{x 4}=J_{y 4}=J_{O 5_{1}}^{(5)}$ then it can be determinate the kinetic energy of the module 5:

$$
\begin{gathered}
E_{c_{5}}=\frac{1}{2}\left(J_{x 4} \omega_{x 5}^{2}+J_{y 4} \omega_{y 5}^{2}+J_{z 4} \omega_{z 5}^{2}\right)+\frac{1}{2} m_{5} v_{05}^{2} \\
E_{c_{5}}=\frac{1}{2} m_{5}\left(\dot{v}_{1}^{2}+\dot{v}_{2}^{2}+\dot{v}_{3}^{2}\right)+\frac{1}{2}\left(J_{O_{5_{1}}}^{(5)}+m_{5} l_{5}^{2}\right) \dot{q}_{2}^{2}+
\end{gathered}
$$




$$
+m_{5}\left(\dot{v}_{2} \dot{v}_{3} \cos q_{2}-\dot{v}_{2} \dot{q}_{2} \sin q_{2}\right)
$$

Given $(3,4,9,14,16)$, the kinetic energy of the TRTTR robot was written:

$$
\begin{aligned}
E_{c}= & \frac{1}{2}\left(\sum_{i=1}^{5} m_{i}\right) \dot{v}_{1}^{2}+\frac{1}{2}\left(\sum_{i=3}^{5} m_{i}\right) \dot{v}_{2}^{2}+\frac{1}{2}\left(\sum_{i=4}^{5} m_{i}\right) \dot{v}_{3}^{2} \\
& +\frac{1}{2}\left[J_{\Delta_{1}}^{(2)}+J_{\Delta_{1}}^{(3)}+m_{3}\left(l_{3}+v_{2}\right)^{2}\right] \dot{q}_{1}^{2}+ \\
+\frac{1}{2}\left[J_{O_{41}}^{(4)}+J_{O_{51}}^{(5)}+\right. & \left.m_{4}\left(l_{4}+v_{3}\right)^{2}+m_{5} l_{5}^{2}\right] \dot{q}_{2}^{2}+m_{3} \dot{v}_{1} \dot{v}_{2} \cos q_{1}-m_{3}\left(l_{3}+v_{2}\right) \dot{v}_{1} \dot{q}_{1} \sin q_{1} \\
& +\left(\sum_{i=4}^{5} m_{i}\right) \dot{v}_{2} \dot{v}_{3} \cos q_{2}-\left[m_{4}\left(l_{4}+v_{3}\right)+m_{5} l_{5}\right] \dot{v}_{2} \dot{q}_{2} \sin _{2} .
\end{aligned}
$$

In (17), the notations were made:

$$
\begin{aligned}
& c_{11}=\left(\sum_{i=1}^{5} m_{i}\right), c_{33}=\left(\sum_{i=3}^{5} m_{i}\right), c_{44}=\left(\sum_{i=4}^{5} m_{i}\right), c_{22}=J_{\Delta_{1}}^{(2)}+J_{\Delta_{1}}^{(3)}+m_{3}\left(l_{3}+v_{2}\right)^{2}, \\
& c_{55}=J_{O_{41}}^{(4)}+J_{O_{51}}^{(5)}+m_{4}\left(l_{4}+v_{3}\right)^{2}+m_{5} l_{5}^{5}, c_{13}=m_{3} \cos q_{1}, \\
& c_{12}=-m_{3}\left(l_{3}+v_{2}\right) \sin q_{1}, c_{34}=\left(\sum_{i=4}^{5} m_{i}\right) \cos q_{2}, \\
& c_{35}=-\left[m_{4}\left(l_{4}+v_{3}\right)+m_{5} l_{5}\right] \sin q_{2} .
\end{aligned}
$$

Therefore (17) becomes:

$$
\begin{aligned}
E_{c}= & \frac{1}{2}\left(c_{11} \dot{v}_{1}^{2}+c_{33} \dot{v}_{2}^{2}+c_{44} \dot{v}_{3}^{2}+c_{22} \dot{q}_{1}^{2}+c_{55} \dot{q}_{2}^{2}+2 c_{13} \dot{v}_{1} \dot{v}_{2}+\right. \\
& +2 c_{12} \dot{v}_{1} \dot{q}_{1}+2 c_{34} \dot{v}_{2} \dot{v}_{3}+2 c_{35} \dot{v}_{2} \dot{q}_{2} .
\end{aligned}
$$

The generalized forces $Q_{k}, k=1 \div 3$ are obtained by applying virtual elementary displacements to the system so that, the generalized coordinates varies in turn, respectively with $\delta v_{1}, \delta v_{2}, \delta v_{3}, \delta q_{1}, \delta q_{2}$. The virtual elementary mechanical work, corresponding to the forces of gravity, mobility, motor moments and some virtual elementary displacements compatible with robotic connections, is:

$$
\begin{aligned}
\delta L= & \left(F_{1}-P_{1}\right) \delta v_{1}+M_{2} \delta \theta_{1}-P_{2} \delta z_{1}+\left(F_{3}-P_{3}\right) \delta r_{2}+ \\
& +\left(F_{4}+P_{4}\right) \delta r_{3}+M_{5} \delta \theta_{4}-P_{5} \delta z_{4}
\end{aligned}
$$

Applying virtual elementary displacements to (20), becomes:

$$
\delta L=\left[F_{1}-\left(\sum_{i=1}^{5} P_{i}\right)\right] \delta v_{1}+M_{2} \delta q_{1}+F_{3} \delta v_{2}+F_{4} \delta v_{3}+M_{5} \delta q_{2} .
$$

By imposing independence conditions on virtual elementary displacements and knowing that $Q_{k}=\frac{\delta L}{\delta q_{k}}, k=1 \div 5$, from (21) the generalized forces can be inferred, with the following equations:

$$
Q_{1}=F_{1}-\left(\sum_{i=1}^{5} P_{i}\right), Q_{2}=M_{2}, Q_{3}=F_{3}, Q_{4}=F_{4}, Q_{5}=M_{5}
$$

Thus, the differential equations of motion of the TRTTR robot were obtained: 


$$
\begin{aligned}
& \left(\sum_{i=1}^{5} m_{i}\right) \ddot{v}_{1}-m_{3} \dot{v}_{1} \dot{v}_{2} \sin q_{1}-m_{3}\left(l_{3}+v_{2}\right) \dot{v}_{1} \dot{q}_{1} \cos q_{1}=F_{1}-\left(\sum_{i=1}^{5} P_{i}\right) \\
& {\left[J_{\Delta_{1}}^{(2)}+J_{\Delta_{1}}^{(3)}+m_{3}\left(l_{3}+v_{2}\right)^{2}\right] \ddot{q}_{1}+m_{3}\left(l_{3}+v_{2}\right) \dot{v}_{1} \dot{q}_{1} \cos q_{1}=M_{2}} \\
& \left(\sum_{i=3}^{5} m_{i}\right) \ddot{v}_{2}-m_{3} \dot{v}_{1} \dot{v}_{2} \sin q_{1}-\left(\sum_{i=4}^{5} m_{i}\right) \dot{v}_{2} \dot{v}_{3} \sin q_{2} \\
& \quad-\left[m_{4}\left(l_{4}+v_{3}\right)+m_{5} l_{5}\right] \dot{v}_{2} \dot{q}_{2} \cos q_{2}=F_{3} \\
& \left(\sum_{i=4}^{5} m_{i}\right) \ddot{v}_{3}-\left(\sum_{i=4}^{5} m_{i}\right) \dot{v}_{2} \dot{v}_{3} \sin q_{2}=F_{4} \\
& {\left[J_{O_{4}}^{(4)}+J_{O_{5_{1}}}^{(5)}+m_{4}\left(l_{4}+v_{3}\right)^{2}+m_{5} l_{5}^{2}\right] \ddot{q}_{2}-\left[m_{4}\left(l_{4}+v_{3}\right)+m_{5} l_{5}\right] \dot{v}_{2} \dot{q}_{2} \cos q_{2}=M_{5} .}
\end{aligned}
$$

The differential equations (23) has been established on the assumption that all robot movements occur simultaneously.

\section{Choosing the driving motor for the MTB SIL module in the mechanical structure of the TRTTR modular serial tracked robot}

\subsection{The constructive solution of MTB SIL module}

In what follows, the constructive solution designed for the horizontal translation module at the base of the modular serial tracked robot will be presented [11]. The module at the base of the robot, which ensures the translational movement along the $y_{0}$ axis, is built on the basis of a screw - nut mechanism, which transforms the rotational motion into a translational one. The main components of the translation module at the base of the robot MTB SIL are presented in Figure 2. The module of rotation along the $z_{0}$ axis and the robot arm are mounted on the flange of the base modulus with ball nut incorporated. Ball screw system is supported at ends by bearings and is driven by an electrical motor which will generate its rotational motion. Thus, due to the ball recirculation system in the nut construction, the rotational motion of the screw is transformed into translational motion along the $y_{0}$ axis of the flange, respectively of the entire structure of the robot. Since the module is required to have a positioning accuracy of under a tenth of a millimeter, it was equipped with a DC motor-driven ball screw, a solution that enables the relatively simple joining of the MTB to a microprocessor-controlled system. Positioning accuracy is also ensured by mounting position encoder onto the end of the ball screw, enabling the movement of heavy loads with high accuracy.

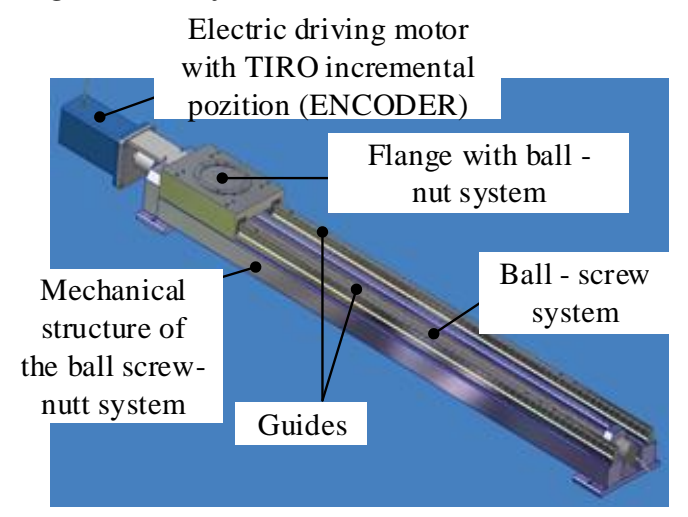

Figure 2. The MTB SIL translation module.

By using ball guides, an increase in the accuracy of motion transmission and a reduction in resistance forces and moments due to the sliding friction are obtained. The MTB 
SIL module, designed and equipped along these lines, can operate in a closed loop with the computer controlling the robot, being adaptable to any types of serial modular robots that are structured on the horizontal translation module from the base (this implicitly leading, based on the equations stated above, to the choice of motors suitable for each robot structure). The position of the movable system is controlled by encoder which is mounted onto the end of the screw through a flexible coupling, having the role to protect the encoder's shaft from the system vibrations and to transmit the screw's rotational movement to the encoder.

\subsection{The mathematical model for choosing the driving motor for the MTB SIL module}

The effective axial force Fa [N] necessary to put the MTB SIL translation module into motion has the following equation $[12,13]$ :

$$
F_{a}=F_{s b .}-F_{f}
$$

where: $F_{s b}$. represents the axial force developed by the ball screw transmission and $F_{f}$ the friction force in the guideway. [12]:

The $F_{s b}$. force developed by the ball screw transmission has the following equation

$$
F_{s b .}=\frac{2 \cdot M_{s}}{d_{0} \cdot \tan \left(\varphi+\arctan \frac{k}{d_{b} \sin \phi}\right)},
$$

where: $d_{0}$ represent the diameter of the cylinder that holds the balls centers, $\varphi$ is the dwell angle of the helix on the medium cylinder, $k$ is the rolling friction coefficient, $d_{b}$ represents the ball diameter, $\phi$ is the contact angle between the ball and the runway, $M_{s}$ represents the torque at the ball screw axle.

By noting with $P_{s,} n_{s}$ the power and the angular speed of the screw ball, the torque $M_{s}$ has the following equation:

$$
\begin{gathered}
M_{s}=9550 \cdot \frac{P_{s}}{n_{s}} \\
P_{s}=P_{m} \cdot \eta_{r} .
\end{gathered}
$$

In (27) $\eta_{r}$ represents the output of a pair of bearings and $P_{m}$ is the power developed by the DC motor that drives the movable system at the robotic base into motion. The angular speed of the screw ball $n_{s}$, must fulfill the following inequality $n_{s} \leq n_{m}$ where by $n_{m}$ it was noted the angular speed of the driving shaft. Given the $(26,27)$ and previous inequality, (25) becomes:

$$
F_{s b .}=\frac{191 \cdot 10^{2} \cdot P_{m} \cdot \eta_{r}}{n_{m} \cdot d_{0} \cdot \tan \left(\varphi+\arctan \frac{k}{d_{b} \sin \phi}\right)}
$$

Having in view (24) and (28), the dynamic equation (23), corresponding to horizontal translation module 1 at the robotic base (MTB SIL), becomes:

$$
\begin{gathered}
\left(\sum_{i=1}^{5} m_{i}\right) \ddot{v}_{1}-m_{3} \dot{v}_{1} \dot{v}_{2} \sin q_{1}-m_{3}\left(l_{3}+v_{2}\right) \dot{v}_{1} \dot{q}_{1} \cos q_{1}= \\
=\frac{191 \cdot 10^{2} \cdot P_{m} \cdot \eta_{r}}{n_{m} \cdot d_{0} \cdot \tan \left(\varphi+\arctan \frac{k}{d_{b} \sin \phi}\right)}-\left(\sum_{i=1}^{5} P_{i}\right)-F_{f} .
\end{gathered}
$$

The friction force $F_{f}$ has the following equation:

$$
F_{f}=\left(\sum_{i=1}^{5} m_{i}\right) \cdot g \cdot \mu,
$$

where by $g$ it was noted the gravitational acceleration $(g=9.8 \mathrm{~m} / \mathrm{s} 2)$ and by $\mu$ the friction coefficient in the guides adopting its standard value $(\mu=0.02)$. 
The dwell angle of the helix on the medium cylinder $(\varphi)$ is calculated using the equation:

$$
\varphi=\operatorname{arctang} \frac{p_{h}}{\pi \cdot d_{0}}
$$

where by $p_{h}$ it was noted the feed screw twist.

Thus, with (30) it was obtained the ratio $\frac{P_{m}}{n_{m}}$ :

$$
\begin{gathered}
{\left[\left(\sum_{i=1}^{5} m_{i}\right) \ddot{v}_{1}-m_{3} \dot{v}_{1} \dot{v}_{2} \sin q_{1}-m_{3}\left(l_{3}+v_{2}\right) \dot{v}_{1} \dot{q}_{1} \cos q_{1}+\right.} \\
\left.+\left(\sum_{i=1}^{5} P_{i}\right)+F_{f}\right] \cdot \frac{d_{0} \cdot \tan \left(\varphi+\arctan \frac{k}{d_{b} \sin \phi}\right)}{191 \cdot 10^{2} \cdot P_{m} \cdot \eta_{r}}=\frac{P_{m}}{n_{m}} .
\end{gathered}
$$

With (34), it can be calculate the motor driving moment, based on which it can be selected the driving motor of the MTB SIL translation module.

\section{Theoretical results}

For TRTTR modular serial tracked robot it was calculated the motor driving moment, $M_{m}$ using the dynamic and organological equations given in the previous sections. For this purpose, the following input data regarding the constructive and functional parameters of the serial tracked robot were taken into account: $m_{1}=41 \mathrm{~kg} ; m_{2}=44.75 \mathrm{~kg} ; \mathrm{m}_{3}=43 \mathrm{~kg} ; \mathrm{m}_{4}$ $=30.41 \mathrm{~kg} ; m_{5}=7.72 \mathrm{~kg} ; l_{3}=0.04 \mathrm{~m} ; p_{h}=0.008 \mathrm{~m} ; d_{0}=0.03 \mathrm{~m} ; \mathrm{k}=0.00001 \mathrm{~m} ; \mathrm{d}_{b}=0.0055 \mathrm{~m} ; \varphi=$ $30^{\circ} ; \eta_{r}=0.995 ; q_{1}=\pi / 4 ; v_{2}=0.25 \mathrm{~m} ; \dot{q}_{1}=0.75 \mathrm{rad} / \mathrm{s} ; \ddot{v}_{1}=1 \mathrm{~m} / \mathrm{s}^{2} ; \dot{v}_{2}=0.4 \mathrm{~m} / \mathrm{s}$. It is specified that the mass values of the robot modules include the mass of the clamping device.

After performing the calculations, the following results were obtained (Table 1):

Table 1. Theoretical results.

\begin{tabular}{|c|c|c|c|}
\hline $\begin{array}{c}\text { Friction force } \\
\mathrm{F}_{\mathrm{f}}[\mathrm{N}]\end{array}$ & $\begin{array}{c}\text { Dwell angle } \\
\boldsymbol{\varphi}\left[^{\circ}\right]\end{array}$ & $\begin{array}{c}\text { Power driving motor } \\
\text { and angular speed ratio } \\
\mathbf{P}_{\mathrm{m}} / \mathbf{n}_{\mathrm{m}}[\mathbf{k W} \cdot \mathbf{m i n} / \mathbf{r o t}]\end{array}$ & $\begin{array}{c}\text { Driving moment } \\
M_{m}[\mathrm{~N} \cdot \mathrm{m}]\end{array}$ \\
\hline 32.708 & 4.852 & $2.02275 \cdot 10^{-4}$ & 1.93 \\
\hline
\end{tabular}

With calculated value of the driving moment, $M_{m}$, it was easily chosen the real diving motor. Thus: $M_{m}=1.93 \mathrm{~N} \cdot \mathrm{m} \rightarrow M_{m_{-} B S}=3.5 \mathrm{~N} \cdot \mathrm{m}$, with the help of which, the DC driving motor it was selected together with the servomotor type 1FT6061-1AF71_A_O from the Siemens NC 60.1-DREHSTROMMOTOREN catalogue with the following characteristics: $M_{m}=3.5 \mathrm{~N} \cdot m ; n_{m}=3000 \mathrm{rpm} ; P_{\text {calc. }}=1.3 \mathrm{~kW} ; m=8.3 \mathrm{~kg}$.

\section{Conclusions}

The tracked robot presented in the invention solves technical issues such as flexibility through the simultaneous accomplishment of several military operations by its modular construction, autonomy by using electric motors powered by solar panels with photovoltaic cells minimizing environmental pollution and energy consumption, reducing assembling and maintenance time due to its modularized compact structure, are used materials and components resistant to dangerous environments preventing the partial or total destruction of the vital mechanical elements in the operation of the tracked robot, it is provided with a storage compartment for the explosive necessary for humanitarian demining, the communication between the human operator and the robot taking place wirelessly, based on a predefined computer program [1,2]. Dynamic modeling using Lagrange's formalism of TRTTR modular serial structure of the tracked robot it was performed in order to obtain the differential equations of motion of the robot which, together 
with the given design equations, lead to the optimal choice of the drive motors of the modules in the robot structure. In this context, the mathematical model for choosing the drive motor for the translation module at the base of the modular robot (MTB SIL module) is given. Also, the mechanical components of the base translation module are briefly presented.

Following the practical realization of the technological product, we formulated the following hypotheses of the scientific research:

- the robotic product will be able to be integrated into demining teams to carry out missions to ensure the mobility of its troops by executing mine corridors through minefields, total demining missions of minefields and humanitarian demining missions;

- the robotic product will be programmed to carry out the entire demining activity autonomously: research, scanning and destruction, and upon discovery of a mine to leave on explosive ground that will detonate the mine buried (or located on the surface of the ground) by sympathy. If it detects UXO ammunition, the robot will mark the ground with paint so that the demining teams can carry out the demining because they cannot be neutralized with a load placed on the ground surface.

\section{Patents}

Petrişor, S.M., Bârsan, Gh., Simion, M., Virca, I., Moşteanu, D.E., "Tracked robot destined for humanitarian demining operations", Invention Patent No. RO 132301B1 / 30.12.2021, no. C.B.I.: a 2017 00562, International Class: F41H11/16, Publication no.: 132301 A0, Deposit date: 10.08.2017, Published C.B.I.: 29/12/2017//12/2017, Published B.I.: 30/12/2021//12/2021, Holder: "Nicolae Bălcescu" Land Forces Academy, Sibiu, C.B.I.: Official Bulletin of Industrial Property, Patent Section, Nr. 12/2017, ISSN 2065-2100, p 18, B.I.: Official Bulletin of Industrial Property, Patent Section, No. 12/2021, ISSN 2065-2100, p. 90, OSIM Bucharest.

Author Contributions: Conceptualization, Petrişor, S.M. and Simion, M.; methodology, Petrişor, S.M.; validation, Petrişor, S.M.; formal analysis, Petrişor, S.M.; investigation, Petrişor, S.M. and Simion, M.; resources, Petrişor, S.M. and Simion, M.; writing-original draft preparation, Petrişor, S.M. and Simion, M.; writing - review and editing, Petrişor, S.M. and Simion, M.; visualization, Petrişor, S.M. and Simion, M.; supervision, Petrişor, S.M.; project administration, Petrişor, S.M.; funding acquisition, Petrişor, S.M..

Funding: This research highlighted in this paper represents disseminations of scientific activities undertaken and funded through the research project of Ministry of National Defense of Romania, position 63\&4/2020-2022, tip PSCD, acronym AVANGARDROBO.

Data Availability Statement: Not applicable here.

Conflicts of Interest: The authors declare no conflict of interest. The funders had no role in the design of the study; in the collection, analyses, or interpretation of data; in the writing of the manuscript, or in the decision to publish the results.

\section{References}

1. Petrişor S.M., Simion M., Example of good practices regarding the organological construction of a robotized technological product for humanitarian engineering operations, Acta Technica Napocensis, Series: Applied Mathematics, Mechanics and Engineering, 2021, Volume 64, Issue 3, ISSN 1221-5872, pp. 395-402.

2. Petrişor, S.M., Simion, M., Aspects Regarding the Elaboration of the Geometric, Kinematic and Organological Study of a Robotic Technological Product "Humanitarian PetSim Robot" Used as an Avant-Garde Element of the Human Factor in High Risk Areas, in Arai K., Kapoor S., Bhatia R. (eds) Intelligent Systems and Applications. IntelliSys 2020, Advances in Intelligent Systems and Computing, 2021, Volume 1250, pp. 322-334.

https://doi.org/10.1007/978-3-030-55180-3_24

3. Benhabib, B., Dai, M.Q., Mechanical design of a modular robot for industrial applications, Journal of Manufacturing Systems, 1991, Volume 10(4), pp 297-306.

4. Fei, Y., Zhao, X., Xu, W. L., Kinematics and dynamics of reconfigurable modular robots, SMC'98 Conference Proceedings, 1998, IEEE International Conference on Systems, Man, and Cybernetics (Cat. No.98CH36218), Volume 4, pp. 3325-3334.

https://doi: 10.1109/ICSMC.1998.726517 
5. Gwiazda, A., Banas, W., Sekala, A., Foit, K., Hryniewicz, P., Kost, G., Modular industrial robots as the tool of process automation in robotized manufacturing cells, IOP Conference, Series: Materials Science and Engineering 95, 2015. doi: 10.1088/1757-899X/95/1/012104

6. Ispas, V., Manipulators and Industrial Robots, Didactic and Pedagogic Publishing House, R.A., Bucharest, 2004.

7. Negrean, I., Kinematics and dynamics of robots: Modeling - experiment - precision, Didactic and Pedagogic Publishing House, R.A., Bucharest, 1999.

8. Drimer, D., et.al., Industrial robots and manipulators, Technical Publishing House, 1985.

9. Petrişor, S.M., Bârsan, G., Aspects on the design, implementation, and simulation of a tracked mini robot destined for special applications in theatres of operations, Proc. SPIE 9067, Sixth International Conference on Machine Vision (ICMV 2013), 90670K, 2013. https://doi.org/10.1117/12.2049918

10. Chircor, M., Curaj, A., Kinematic, Dynamic Elements and Trajectory Planning of Industrial Robots, The Publishing House of the Romanian Academy, Bucharest, 2001.

11. Petrişor, S.M., Industrial Robots Used in Special Applications, The „Nicolae Bălcescu” Land Forces Academy Publishing House, Sibiu, 2010.

12. Chişu, A., et.al., Machine Parts. Didactic and Pedagogic Publishing House Bucharest, R.A., 1981.

13. Petrişor, S.M., Bârsan, G., Aspects on the calculus and construction of translation modules in the mechanical structure of an industrial robot possessing four degrees of freedom, Journal of Applied Mechanics and Materials - Mechanical and Aerospace Engineering IV, 2013, Volume 390, pp. 166-171.

https://doi.org/10.4028/www.scientific.net/AMM.390.166 